For reprint orders, please contact: reprints@futuremedicine.com

\section{Randomized, double-blind study comparing proposed biosimilar LA-EP2006 with reference pegfilgrastim in breast cancer}

\author{
Nadia Harbeck*, , Oleg Lipatov ${ }^{2}$, Mona Frolova ${ }^{3}$, Dmitry Udovitsa ${ }^{4}$, Eldar Topuzov ${ }^{5}$, \\ Doina Elena Ganea-Motan ${ }^{6}$, Roumen Nakov ${ }^{7}$, Pritibha Singh 7 , Anita Rudy ${ }^{7}$ \\ \& Kimberly Blackwell ${ }^{8}$
}

\begin{abstract}
Aim: This randomized, double-blind trial compared proposed biosimilar LA-EP2006 with reference pegfilgrastim in women receiving chemotherapy for breast cancer (PROTECT-1). Patients \& methods: Women ( $\geq 18$ years) were randomized to receive LA-EP2006 $(n=159)$ or reference $(n=157)$ pegfilgrastim (Neulasta ${ }^{\oplus}$, Amgen) for $\leq 6$ cycles of (neo)-adjuvant TAC chemotherapy. Primary end point was duration of severe neutropenia (DSN) during cycle 1 (number of consecutive days with absolute neutrophil count $<0.5 \times 10^{9} /$ l) with equivalence confirmed if $90 \%$ and $95 \% \mathrm{Cls}$ were within a \pm 1 day margin. Results: For DSN, LA-EP2006 was equivalent to reference (difference: 0.07 days; $90 \% \mathrm{Cl}:-0.09-0.23 ; 95 \% \mathrm{Cl}:-0.12-0.26$ ). Conclusion: LA-EP2006 and reference pegfilgrastim showed no clinically meaningful differences regarding efficacy and safety in breast cancer patients receiving chemotherapy.
\end{abstract}

First draft submitted: 8 January 2016; Accepted for publication: 1 March 2016; Published online: 29 March 2016

Cancer represents a substantial burden on healthcare systems, with escalating drug costs a major contributory factor [1]. Biosimilars are approved biologics in highly regulated markets that have been proven to be highly similar to a reference product with no meaningful differences in clinical performance. Biosimilars offer the potential to allow increased access to biological treatments [2].

The development of biosimilars follows a step-wise approach including analytical comparison to the reference and iterative process development to achieve a product which is essentially the same as the reference product [3]. The clinical trials in support of this step-wise process are focused on confirming this similarity so that the totality of data reinforce that the biosimilar is essentially the same biological substance as the reference product [4]. The clinical trial reported below was conducted to confirm the similarity of a proposed biosimilar to commercial pegfilgrastim.

The recombinant human granulocyte-colony-stimulating factor (G-CSF), filgrastim and its longacting pegylated form, pegfilgrastim, are widely used for the prevention of chemotherapy-induced neutropenia. Biosimilars of filgrastim, based on the reference product Neupogen ${ }^{\circledR}$, have been licensed in Europe for over 6 years and, in 2015, filgrastim $\left(\right.$ Zarxio $\left.^{\circledR}\right)$ became the first biosimilar product approved by the US FDA in the USA [5].

'Breast Center \& CCCLMU, University of Munich, Munich, Germany

${ }^{2}$ Republican Clinical Oncology Dispensary of the Ministry of Public Health of Bashkortostan Republic, Ufa, Russia

${ }^{3}$ Russian Oncology Research Center n.a. N.N. Blochin of RAMS, Moscow, Russia

${ }^{4}$ Oncological Dispensary \#2 of Healthcare Department of Krasnodar Territory, Krasnodar, Russia

${ }^{5}$ Northwest State Medical University n.a. I.I. Mechnikov, St Petersburg, Russia

${ }^{6}$ Spitalul Judetean de Urgenta, Suceava, Romania

${ }^{7}$ Hexal AG (a Sandoz company), Holzkirchen, Germany

${ }^{8}$ Duke University, DUMC, NC, USA

*Author for correspondence: Tel.: +49 0894400 77581; Fax: +49 0894400 77581; nadia.harbeck@med.uni-muenchen.de

\section{KEYWORDS}

- biosimilars • granulocytecolony-stimulating factor

- pegfilgrastim 
G-CSF guidelines recommend primary G-CSF prophylaxis for patients at greater than $20 \%$ risk for $\mathrm{FN}$, including patients receiving TAC chemotherapy [6,7]. Several studies have suggested that the use of daily filgrastim may be suboptimal, with treatment started later and/or dosed for a shorter duration than recommended [6]. Compared with filgrastim, the pegylation of filgrastim results in reduced renal clearance and greater stability [8]. Pegfilgrastim has comparable efficacy and safety to filgrastim, but its longer serum half-life allows once-percycle instead of daily administration $[9,10]$. The greater convenience of once-per-cycle administration may result in better compliance and improved outcomes with pegfilgrastim compared with filgrastim, including reduced incidence of febrile neutropenia (FN) and a lower risk of hospitalization $[11,12]$. However, a trend for superiority of pegfilgrastim compared with filgrastim was reported in some analyses [13].

This global, prospective randomized, doubleblind, multicenter confirmatory efficacy and safety study, PROTECT-1, was a head-to-head comparison of a proposed biosimilar pegfilgrastim (LA-EP2006) with reference pegfilgrastim $\left(\right.$ Neulasta $\left.^{\circledR}\right)$. The study was designed to show equivalence of LA-EP2006 to the reference pegfilgrastim in the reduction of duration of severe neutropenia (DSN) in breast cancer patients receiving myelosuppressive chemotherapy.

\section{Patients \& methods}

PROTECT-1 enrolled patients with breast cancer receiving (neo)-adjuvant myelosuppressive chemotherapy (EudraCT number 2011-00453258). The study was conducted in accordance with ICH Guidelines for Good Clinical Practice, the Declaration of Helsinki, and local regulations. The study protocol was approved by Independent Ethics Committees for each center. All patients provided written informed consent.

Adult women (aged $\geq 18$ years) with histologically proven breast cancer who were eligible for either adjuvant or neoadjuvant chemotherapy with docetaxel $75 \mathrm{mg} / \mathrm{m}^{2}$, doxorubicin $50 \mathrm{mg} / \mathrm{m}^{2}$ and cyclophosphamide $500 \mathrm{mg} / \mathrm{m}^{2}$ (TAC regimen) were enrolled. Inclusion criteria were: Eastern Cooperative Oncology Group (ECOG) performance status $\leq 2$; adequate bone marrow function at day 1 of cycle 1 before chemotherapy (absolute neutrophil count $[\mathrm{ANC}] \geq 1.5 \times 10^{\%} / 1$, platelet count $\geq 100 \times 10^{9} / \mathrm{l}$, hemoglobin $\geq 10 \mathrm{~g} / \mathrm{dl}$ ); normal total bilirubin; aspartate aminotransferase
(AST) and alanine aminotransferase (ALT) level $\leq 2 \times$ upper limit of normal (ULN); liver-derived alkaline phosphatase level $\leq 3 \times \mathrm{ULN}$; creatinine $\leq 1.5 \times$ ULN and for women of child-bearing potential, a negative pregnancy test within 7 days before randomization and use of an effective method of birth control. Patients were excluded for reasons including: history of chronic myeloid leukemia or myelodysplastic syndrome; history of sickle cell disease; previous or concurrent malignancies; any significant relevant medical condition and concurrent or prior anti-breast cancer treatment.

\section{Design}

After a screening period of up to 21 days, eligible patients were randomized 1:1 to either proposed biosimilar pegfilgrastim (LA-EP2006, Sandoz GmbH, Kundl, Austria) or Neulasta (Amgen BV, The Netherlands). Randomization was stratified by chemotherapy (adjuvant or neoadjuvant) and region (Europe, Asia or America). TAC chemotherapy was administered intravenously on day 1 of each chemotherapy cycle and given every 3 weeks for up to six cycles. Chemotherapy doses could be reduced by $25 \%$ in response to grade 3-4 nonhematological toxicity, grade 4 thrombocytopenia or FN. Pegfilgrastim (LA-EP2006 or reference) was administered as a $6 \mathrm{mg}$ subcutaneous injection $(0.6 \mathrm{ml}$ in prefilled single-use syringes) on day 2 of each chemotherapy cycle ( $\geq 24 \mathrm{~h}$ after the end of chemotherapy). Patients were followed for a 6-month safety period from the last administration of pegfilgrastim.

\section{- End points}

The primary efficacy end point was the mean DSN during cycle 1 of chemotherapy, defined as the number of consecutive days with an ANC $<0.5 \times 10^{9} / 1$ (grade 4 neutropenia). Secondary efficacy parameters were depth of ANC nadir (lowest ANC) and time to ANC recovery (days from ANC nadir until ANC increased to $\geq 2 \times 10^{9} / \mathrm{l}$ ) during cycle 1 , incidence of $\mathrm{FN}$ (oral temperature of $\geq 38.3^{\circ} \mathrm{C}$ with $\mathrm{ANC}<0.5 \times 10^{\circ} / \mathrm{l}$ ) or neutropenic sepsis (FN/NS) by cycle and across all cycles, number of patients with fever (oral temperature $\geq 38.3^{\circ} \mathrm{C}$ ) for each cycle, number of patients with infections by cycle and across all cycles, and mortality due to infection.

Safety was assessed through the incidence, occurrence and severity of treatment-emergent adverse events (TEAEs), using Common Terminology Criteria for Adverse Events 
(CTCAE) version 4.0. According to the study protocol, every FN episode was reported as a serious adverse event (AE). Safety assessments were performed at each visit with safety follow-up visits at 4 weeks and 6 months after the last administration of pegfilgrastim. Immunogenicity of pegfilgrastim was assessed by a validated ELISA for screening and confirmation of binding anti-pegfilgrastim antibodies, and a validated cell-based assay for the detection of neutralizing antibodies (NABs). Immunogenicity was assessed before the first administration of pegfilgrastim (LA-EP2006 or reference), on day 15 of cycle 6 , and 4 weeks and 6 months after last administration.

\section{- Statistical analysis}

A sample size of 302 patients was considered sufficient to achieve $90 \%$ power for testing of equivalence (two one-sided tests) at the $2.5 \%$ significance level, assuming no difference in mean DSN between treatments with a common standard deviation (SD) of 1.6 days. The primary efficacy end point was analyzed using analysis of covariance (ANCOVA), adjusted for treatment group, region, chemotherapy and baseline ANC count. Equivalence for the difference in the mean DSN was assessed with both two-sided $90 \%$ CIs (required by the FDA) and 95\% CIs (required by the EMA), with LA-EP2006 considered equivalent to reference pegfilgrastim if CIs were entirely within \pm 1 day. All secondary efficacy end points and safety parameters were descriptively analyzed. All statistical analyses were performed using SAS (SAS Institute, NC, USA).

The full analysis set (FAS) included all randomized patients who received $\geq 1$ dose of pegfilgrastim with patients analyzed according to randomized treatment allocation. The per-protocol (PP) set included all patients who completed the first cycle without major protocol deviation in cycle 1 . The safety analysis (SAF) set included all patients who received $\geq 1$ dose of pegfilgrastim and had $\geq 1$ post-baseline safety assessment.

\section{Results}

The study was conducted between June 2012 and February 2014 at 42 study sites. A total of 373 patients were screened, of whom 316 patients were randomized to treatment and included in the FAS set (LA-EP2006, $\mathrm{n}=159$; reference, $\mathrm{n}=157$ ). The PP set included 295 patients (LA-EP2006, $n=146$; reference, $n=149$ ). A total of 19 patients discontinued treatment in the LA-EP2006 group compared with seven patients in the reference group. The main reason for this was 'withdrawn consent' (eight vs two patients). Disposition of patients is shown in Figure 1. Baseline characteristics of patients were balanced across treatment groups (Table 1).

\section{- Primary efficacy end point}

The primary efficacy variable mean $( \pm S D)$ DSN during cycle 1 was $0.75 \pm 0.88$ days (median, range: $1,0-3$ ) with LA-EP2006 and $0.83 \pm 0.90$ days (median, range: $1,0-4$ ) with reference pegfilgrastim (FAS). The difference between LA-EP2006 and reference pegfilgrastim was 0.07 days ( $90 \%$ CI: $-0.09-0.23$; $95 \% \mathrm{CI}$ : $-0.12-0.26)$. Both the $90 \%$ and $95 \%$ CIs were within the predefined margin of \pm 1 day confirming equivalence. Similar results were seen in the PP population (mean DSN: LA-EP2006 $0.75 \pm 0.88$ days, reference $0.79 \pm 0.87$ days; treatment difference 0.04 days [90\% CI: $-0.12-0.20$; 95\% CI: $-0.15-0.24]$ ) (Table 2).

\section{- Secondary efficacy end points}

No clinically meaningful differences between treatment arms were observed. Mean $( \pm S D)$ depth of ANC nadir in cycle 1 was $1.10 \pm 1.54 \times 10^{9} / 1$ (median, range: $0.56,0.0-8.6$ ) in the LA-EP2006 group and $0.92 \pm 1.18 \times 10^{9} / 1$ (median, range: 0.46 , $0.0-6.9)$ in the reference group, with most cases recorded on day 7. Mean number $( \pm S D)$ of days to ANC recovery was also similar for patients in the LA-EP2006 and reference groups $(1.58 \pm 1.05$ vs $1.72 \pm 1.10$; median of 2 days in both groups [LA-EP2006 range: $0.0-4.0$; reference range: $0.0-5.0]$ ). Time course of mean ANC was almost superimposable for both groups (Figure 2).

In cycle 1 and across all cycles, fewer patients treated with LA-EP2006 compared with reference pegfilgrastim experienced at least one event of FN/NS (cycle $1: \mathrm{n}=6[3.8 \%]$ vs 11 [7.0\%]; all cycles, $\mathrm{n}=9[5.7 \%]$ vs $12[7.6 \%])$. The incidence of fever (cycle $1: \mathrm{n}=9$ [5.7\%] vs 14 [8.9\%]; all cycles, $\mathrm{n}=26[16.4 \%]$ vs 26 [16.6\%]) and frequency of infections (cycle 1: $\mathrm{n}=7$ [4.4\%] vs 4 [2.5\%]; all cycles, $\mathrm{n}=22$ [13.8\%] vs 24 [15.3\%]) were similar in both the LA-EP2006 and reference groups. Two patients, both treated with reference pegfilgrastim, died due to infections (Table 3).

\section{- Chemotherapy relative dose intensity}

Relative mean $( \pm S D)$ dose intensity of the chemotherapy was similar between groups across cycles: docetaxel: LA-EP2006, $0.99 \pm 0.025$, reference: $0.98 \pm 0.048$; doxorubicin: LA-EP2006, 


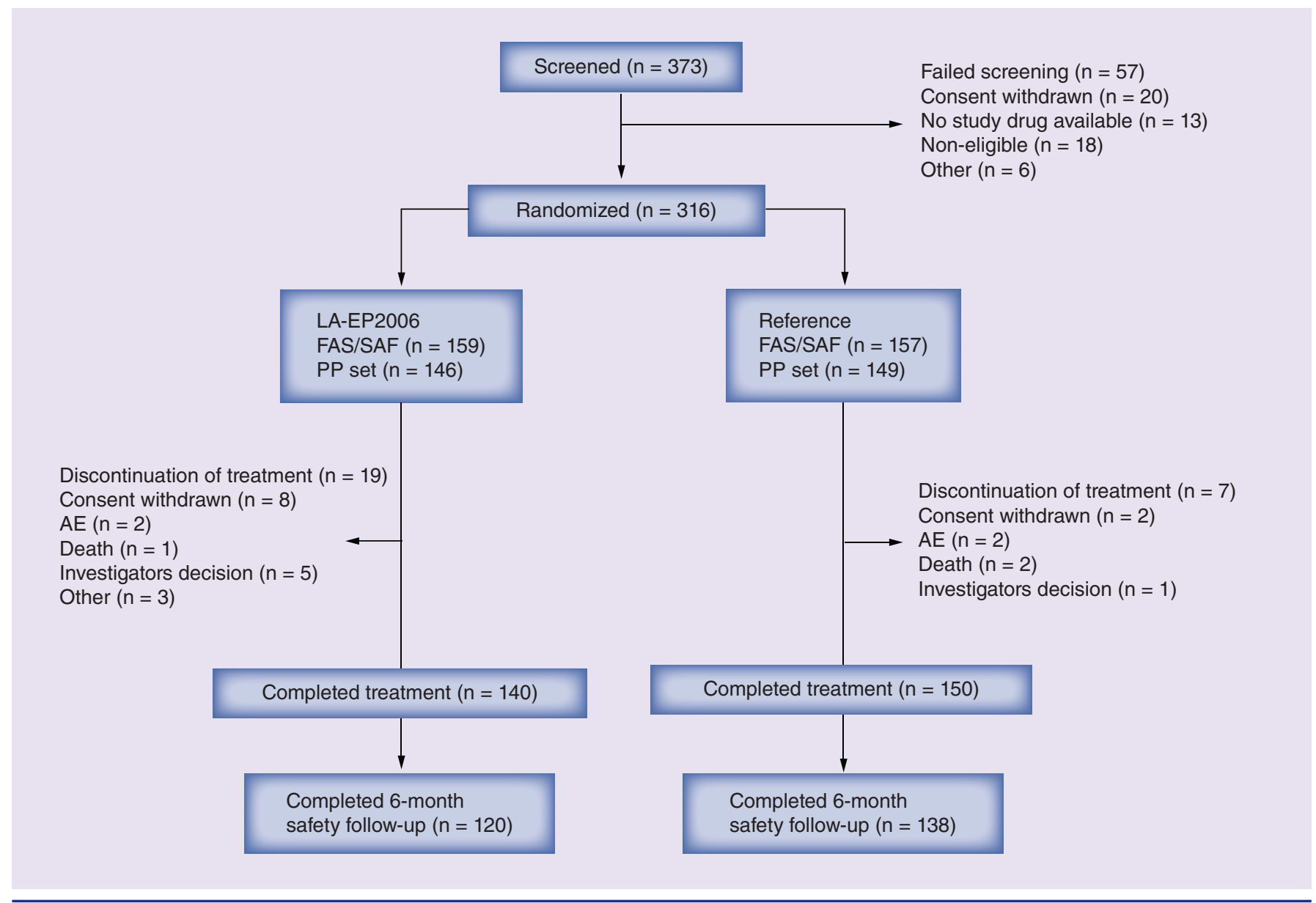

Figure 1. Patient disposition.

AE: Adverse event; FAS: Full analysis set; PP: Per-protocol; SAF: Safety analysis.

$0.99 \pm 0.022$, reference: $0.99 \pm 0.040$; cyclophosphamide: LA-EP2006, $0.99 \pm 0.022$, reference: $0.99 \pm 0.039$.

\section{- Safety}

A total of 88.1 and $82.8 \%$ of patients receiving LA-EP2006 or reference pegfilgrastim experienced $\geq 1$ TEAE during the treatment period (i.e., date of onset of or after the first administration of chemotherapy and not later than 30 days after last pegfilgrastim administration) (Supplementary Figure 1). Type and frequency of TEAEs were similar with LA-EP2006 and reference pegfilgrastim, with the most frequent being alopecia (51.6 vs $50.3 \%$ ), nausea (40.9 vs $37.6 \%$ ), asthenia (39.6 vs $35.7 \%$ ), vomiting (21.4 vs $21.7 \%$ ) and neutropenia (17.0 vs $21.7 \%$ ). TEAEs in the system organ class musculoskeletal and connective tissue disorders (including bone pain, arthralgia, myalgia, pain in extremity, back pain and neck pain) were reported in $15.1 \%$ of patients in the LA-EP2006 group and $22.9 \%$ of patients in the reference group, all of which were grade 1 or 2 in severity. A total of 42 patients (LA-EP2006 11.9\%, $\mathrm{n}=19$; reference $14.6 \%$, $\mathrm{n}=23$ ) had TEAEs with suspected relationship to pegfilgrastim. Serious TEAEs reported in $\geq 2 \%$ of patients in either treatment group were FN (LA-EP2006: 5.7\%, reference: $7.6 \%$ ) and neutropenia (1.9 vs 3.8\%). Six patients reported TEAEs in the 6-month safety follow-up period (LA-EP2006: 2.5\%, reference: 1.3\%), none of which were serious.

FN was reported as a serious TEAE with suspected relationship to pegfilgrastim in three patients in the LA-EP2006 group; two of these patients also had neutropenic sepsis. No patient in the reference group had any serious pegfilgrastim-related TEAE. The incidence of serious neutropenic events are reported in Table 4.

Six deaths occurred during the study, none of which were suspected to be related to pegfilgrastim. 


\begin{tabular}{|c|c|c|}
\hline Characteristic & LA-EP2006 ( $=159)$ & Reference $(\mathrm{N}=157)$ \\
\hline Age, years (mean $\pm S D)$ & $49.9 \pm 9.5$ & $50.5 \pm 10.9$ \\
\hline \multicolumn{3}{|l|}{ Race (n): } \\
\hline - White & 129 & 127 \\
\hline - Asian & 28 & 26 \\
\hline - Other & 2 & 4 \\
\hline $\mathrm{BMI}($ mean $\pm \mathrm{SD}), \mathrm{kg} / \mathrm{m}^{2}$ & $27.5 \pm 26.8$ & $27.4 \pm 26.4$ \\
\hline Time since diagnosis, months; median (range) & $1.35(0.1-76.0)$ & $1.38(0.2-10.9)$ \\
\hline \multicolumn{3}{|l|}{ Disease stage, $\mathrm{n}(\%)$ : } \\
\hline-1 & $4(2.5)$ & $3(1.9)$ \\
\hline$-\|$ & $74(46.5)$ & $73(46.5)$ \\
\hline- III & $81(50.9)$ & $78(49.7)$ \\
\hline$-I V$ & 0 & $3(1.9)$ \\
\hline Previous breast cancer surgery, n (\%) & $149(93.7)$ & $146(93.0)$ \\
\hline Previous radiotherapy, $\mathrm{n}(\%)$ & $7(4.4)$ & $9(5.7)$ \\
\hline ECOG performance status 0/1, $\mathrm{n}(\%)$ & $128(80.5) / 31(19.5)$ & $123(78.3) / 34(21.7)$ \\
\hline \multicolumn{3}{|c|}{$\begin{array}{l}\text { 'Other' race patients }(n=6) \text { were of Mestizo or Parda origin. Time of initial diagnosis missing for six patients in LA-EP2006 group and } \\
\text { ten patients in reference group. } \\
\text { ECOG: Eastern Cooperative Oncology Group; N: Number of patients in a treatment group or analysis set; } n \text { : Number of evaluable } \\
\text { patients; SD: Standard deviation. }\end{array}$} \\
\hline
\end{tabular}

Four deaths occurred in the LA-EP2006 group and two in the reference group.

No neutralizing antibodies were detected in any patient at any time point during the study. Binding antibodies against pegfilgrastim and polyethylene glycol (PEG) were detected in 20 patients (LA-EP2006, $\mathrm{n}=7$; reference, $\mathrm{n}=13$ ) before treatment (on day 1 of cycle 1) but only one patient (LA-EP2006 arm) was positive for anti-pegfilgrastim/PEG antibodies at the end of treatment and another patient (LA-EP2006 arm, tested positive at day 1 , cycle 1 ) was positive at the end of 6-month follow-up.

\section{Discussion}

In this global, prospective, randomized study, LA-EP2006 was shown to be equivalent to reference pegfilgrastim $\left(\right.$ Neulasta $\left.^{\circledR}\right)$, with a difference in DSN between treatments of 0.07 days (90\% CI: -0.09-0.23; 95\% CI: -0.12-0.26). The DSN observed in both groups ( 0.75 days with LA-EP2006 and 0.83 days with reference) was lower than observed in earlier studies of reference pegfilgrastim (mean DSN of 1.3-1.8 days) $[9-10,14]$ but is consistent with more recent studies which reported mean DSNs of 0.8-0.9 days with pegfilgrastim $[15,16]$. This was one of two similar studies comparing LA-EP2006 with reference pegfilgrastim in patients with breast cancer (PROTECT-1 and -2). Equivalence to the reference product was also shown in the PROTECT-2 study [17].

There were also no clinically meaningful differences between LA-EP2006 and reference pegfilgrastim in any of the secondary end points. Incidence of $\mathrm{FN}$ was consistent with previous

Table 2. Primary efficacy parameter: duration of severe neutropenia in cycle 1 (days; full analysis set and per-protocol).

\begin{tabular}{|c|c|c|c|c|}
\hline \multirow[t]{2}{*}{ DSN } & \multicolumn{2}{|c|}{ FAS } & \multicolumn{2}{|c|}{ PP } \\
\hline & LA-EP2006 $(N=159)$ & Reference $(N=157)$ & LA-EP2006 $(N=146)$ & Reference $(N=149)$ \\
\hline $\mathrm{n}$ & $155+$ & $155+$ & 146 & 149 \\
\hline Mean $\pm S D$, days & $0.75 \pm 0.878$ & $0.83 \pm 0.898$ & $0.75 \pm 0.875$ & $0.79 \pm 0.872$ \\
\hline Median (range); days & $1(0-3)$ & $1(0-4)$ & $1(0-3)$ & $1(0-3)$ \\
\hline \multicolumn{5}{|l|}{ Inferential test results of ANCOVA } \\
\hline \multirow[t]{3}{*}{ Treatment difference: reference LA-EP2006 (days) } & 0.07 & & 0.04 & \\
\hline & $90 \% \mathrm{Cl}:-0.09-0.23$ & & $90 \% \mathrm{Cl}:-0.12-0.20$ & \\
\hline & $95 \% \mathrm{Cl}:-0.12-0.26$ & & $95 \% \mathrm{Cl}:-0.15-0.24$ & \\
\hline
\end{tabular}




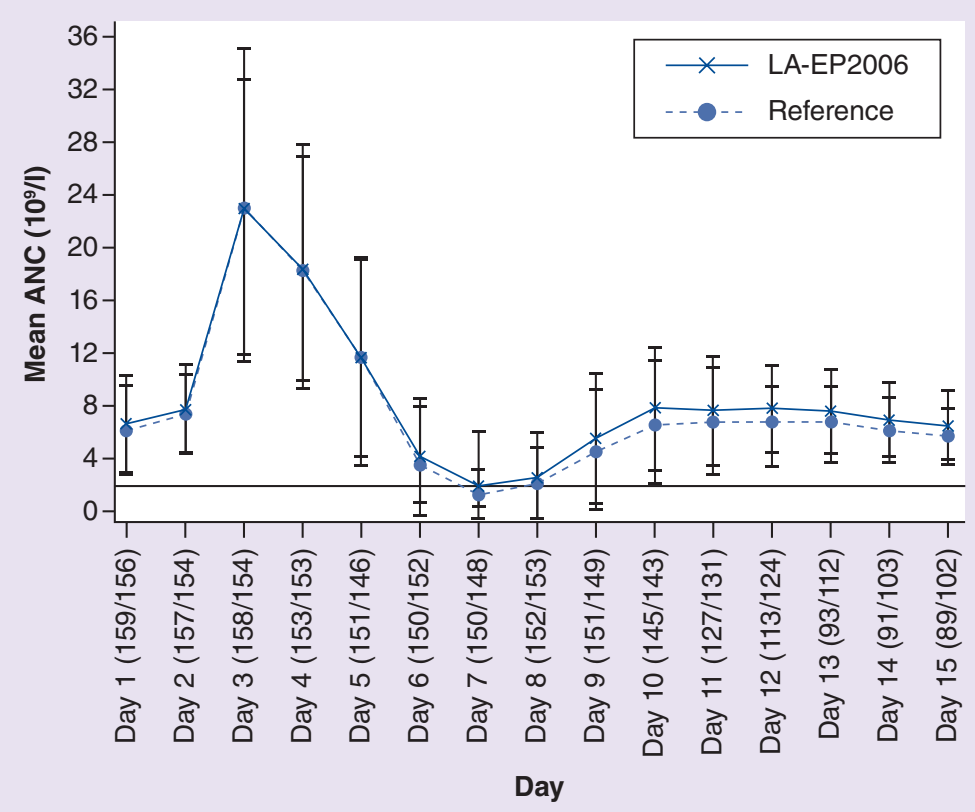

Figure 2. Absolute neutrophil count time course during cycle 1 (mean \pm standard devation; full analysis set).

The horizontal line indicates the threshold of $2 \times 10^{9} /$ I defined for ANC recovery. ANC: Absolute neutrophil count.

studies of reference pegfilgrastim [9-10,14]. The numbers of patients with episodes of fever or with infections were similar in both groups.

Both treatments were well tolerated with a safety profile as expected for patients with breast cancer receiving TAC chemotherapy [9-10,14]. Pegfilgrastim-related TEAEs were similar in both groups and consistent with the known safety profile of pegfilgrastim. The incidence of TEAEs in the system organ class 'musculoskeletal and connective tissue disorders' (15.1\% in patients receiving LA-EP2006 and 22.9\% in patients receiving reference) in this study was slightly lower than in previously randomized double-blind studies with pegfilgrastim which have reported bone pain in the range $25-37 \%$ with pegfilgrastim $[9-10,18]$.

Although fewer patients experienced $\mathrm{FN}$ in the LA-EP2006 group (nine vs 12 patients in the reference group across all cycles), more episodes in the LA-EP2006 group were considered to be pegfilgrastim-related (three patients, two of whom also had neutropenic sepsis versus no patients in the reference group).

None of the six deaths occurring during the study were suspected to be related to pegfilgrastim. In the LA-EP2006 arm, one patient experienced FN as a result of chemotherapy, and deteriorated into respiratory distress leading to cardiorespiratory arrest. Another patient in the LA-EP2006 arm died due to cardiorespiratory arrest. This patient had experienced severe vomiting which, together with poor food intake (over several days) and antidiabetic treatment, may have caused hypoglycemia and cardiorespiratory arrest. The third patient in the LA-EP2006 arm died due to cardiac arrest. A further patient in the LA-EP2006 arm died due to disease progression during the 6-month safety follow-up. Two patients in the reference pegfilgrastim group died, both due to infectious disease.

Immunogenicity can be a concern for any biological product and may occur in response to even seemingly small product-related differences, for example, structural alterations or impurities [19]. No neutralizing anti-pegfilgrastim antibodies were detected in the study, including at the end of the 6-month safety follow-up, indicating that LA-EP2006 had no increased immunogenic potential compared with reference pegfilgrastim. This would be expected given the low immunogenic potential of pegfilgrastim and the immunocompromised status of patients receiving myelosuppressive chemotherapy. Binding antibodies against PEG were detected in 20 patients before treatment. The common use of PEGylated 


\section{Table 3. Secondary efficacy parameters (full analysis set).}

\begin{tabular}{|c|c|c|}
\hline Secondary efficacy parameter & LA-EP2006 ( $=159)$ & Reference $(\mathrm{N}=157)$ \\
\hline Depth of ANC nadir $\left(\times 10^{9} / \mathrm{I}\right)$ in cycle 1 , mean \pm SD & $1.102 \pm 1.5398$ & $0.921 \pm 1.1771$ \\
\hline Time to ANC recovery in cycle 1 , mean \pm SD (median) & $1.58 \pm 1.053(2.0)$ & $1.72 \pm 1.100(2.0)$ \\
\hline \multicolumn{3}{|l|}{ Patients with $\geq 1$ episode of FN/NS, $n(\%)^{+}$: } \\
\hline - Cycle 1 & $6(3.8)$ & $11(7.0)$ \\
\hline - All cycles & $9(5.7)$ & $12(7.6)$ \\
\hline \multicolumn{3}{|l|}{ Patients with $\geq 1$ episode of fever, $n(\%)^{+}$: } \\
\hline - Cycle 1 & $9(5.7)$ & $14(8.9)$ \\
\hline - All cycles & $26(16.4)$ & $26(16.6)$ \\
\hline \multicolumn{3}{|l|}{ Patients with $\geq 1$ infection, $\mathrm{n}(\%)^{\dagger}$ : } \\
\hline - Cycle 1 & $7(4.4)$ & $4(2.5)$ \\
\hline - All cycles & $22(13.8)$ & $24(15.3)$ \\
\hline Mortality due to infection, $\mathrm{n}(\%)$ & 0 & $2(1.3)$ \\
\hline
\end{tabular}

products in cosmetics may have resulted in preexisting anti-PEG antibodies and could explain the higher incidence of positive anti-pegfilgrastim antibodies on day 1 of cycle 1 . As with other biological drugs, ongoing post-authorization surveillance after marketing authorization of biosimilar products is required to confirm the lack of an immunogenic response.

\section{Future perspective}

Cancer represents a substantial burden on healthcare systems. Development of oncology biosimilars such as biosimilar pegfilgrastim could benefit patients by increasing access to biologic agents which has the potential to improve clinical outcomes. An example of this was shown at two hospitals, one in Germany and another in the UK, where the decision to switch from using reference filgrastim to Zarzio ${ }^{\circledR}$ contributed to a shift in treatment practice from secondary prophylaxis to increased primary prophylaxis [20]. This experience suggests that availability of LA-EP2006 may allow preventative treatment of patients, rather than just after they have experienced neutropenic complications. MONITOR-GCSF was an international, multicenter observational study of cancer patients treated with myelosuppressive chemotherapy and receiving prophylaxis with biosimilar filgrastim (Zarzio) [21]. The study showed that in real-world use, clinical and safety outcomes of prophylaxis with biosimilar filgrastim are similar to historical data for reference filgrastim and highlighted the need to improve primary prophylaxis in patients with cancer who are at risk of FN. Biosimilars such as LA-EP2006 may become widely accepted and used, facilitating access to such supportive care treatment for patients.

Table 4. Incidence of serious neutropenic events by category and preferred term (safety analysis set).

\begin{tabular}{|c|c|c|}
\hline Severe neutropenic event & LA-EP2006 ( $=159), n(\%)$ & Reference ( $\mathrm{N}=157), \mathrm{n}(\%)$ \\
\hline \multicolumn{3}{|c|}{ Patients with $\geq 1$ serious neutropenic event } \\
\hline Febrile neutropenia & $9(5.7)$ & $12(7.6)$ \\
\hline Neutropenia & $3(1.9)$ & $6(3.8)$ \\
\hline Neutropenic sepsis & $2(1.3)$ & 0 \\
\hline Leukopenia & 0 & $1(0.6)$ \\
\hline \multicolumn{3}{|c|}{ Patients with $\geq 1$ serious neutropenic event with suspected relationship to pegfilgrastim } \\
\hline Febrile neutropenia & $3(1.9)$ & 0 \\
\hline Neutropenic sepsis & $2(1.3)$ & 0 \\
\hline
\end{tabular}




\section{Conclusion}

LA-EP2006 was equivalent to reference pegfilgrastim in efficacy and safety in the prevention of neutropenia in patients with breast cancer receiving TAC chemotherapy.

\section{Supplementary data}

To view the supplementary data that accompany this paper please visit the journal website at: http://www.futuremedicine.com/doilfull/10.2217/fon-2016-0016.

\section{Acknowledgements}

For a full list of the PROTECT-1 trial investigators, please see the Supplementary Information.

\section{Disclosure}

The study number (EudraCT no.) is 2011-004532-58.

Financial \& competing interests disclosure This work was supported by Sandoz GmbH, Kundl, Austria. $N$ Harbeck has provided advisory or consulting services to Amgen and Sandoz GmbH, Kundl, Austria. K Blackwell has provided advisory or consulting services to Sandoz GmbH, Kundl, Austria. M Frolova has provided advisory or consulting services to Astra Zeneca, Biocad,
Roche, Sanofi and Sandoz GmbH, Kundl, Austria. R

Nakov, P Singh and A Rudy are employees of Hexal AG (a Sandoz company), Holzkirchen, Germany. The authors have no other relevant affiliations or financial involvement with any organization or entity with a financial interest in or financial conflict with the subject matter or materials discussed in the manuscript apart from those disclosed.

Editorial support was provided by Andy Bond and Caroline McGown of Spirit Medical Communications Ltd, supported by Sandoz GmbH, Kundl, Austria.

\section{Ethical conduct of research}

The authors state that they have obtained appropriate institutional review board approval or have followed the principles outlined in the Declaration of Helsinki for all human or animal experimental investigations. In addition, for investigations involving human subjects, informed consent has been obtained from the participants involved.

\section{Open access}

This work is licensed under the Creative Commons Attribution-NonCommercial 4.0 Unported License. To view a copy of this license, visit http://creativecommons.org/ licenses/by-nc-nd/4.0/

\section{EXECUTIVE SUMMARY}

\section{Background}

- Pegfilgrastim is widely used for the prevention of chemotherapy-induced neutropenia.

- Biosimilars offer the potential to increase access to biological treatments.

\section{Results}

- In women with breast cancer receiving TAC chemotherapy, LA-EP2006 was equivalent to reference pegfilgrastim in terms of duration of severe neutropenia in cycle 1.

- Treatment-emergent adverse events were similar across groups and no anti-pegfilgrastim neutralizing antibodies were detected.

\section{Discussion}

- LA-EP2006 met the primary end point, demonstrating equivalence to the reference.

- LA-EP2006 and reference pegfilgrastim are similar with no clinically meaningful differences regarding efficacy and safety in breast cancer patients receiving (neo)-adjuvant chemotherapy.

\section{References}

Papers of special note have been highlighted as: - of interest; $\bullet$ of considerable interest

1 National Cancer Policy Forum. Ensuring Patient Access to Affordable Cancer Drugs: Workshop Summary. Board on Health Care Services, Institute of Medicine (Eds). National Academies Press, Washington, DC, USA (2014).
2 Bennett CL, Chen B, Hermanson T et al. Regulatory and clinical considerations for biosimilar oncology drugs. Lancet Oncol. 15(13), e594-e605 (2014).

3 McCamish M, Woollett G. The state of the art in the development of biosimilars. Clin. Pharmacol. Ther. 91(3), 405-417 (2012).

4 European Medicines Agency. Questions and Answers on Biosimilar Medicines (Similar
Biological Medicinal Products). EMA/837805/2011. EMA, London, 2012.

5 Blackwell K, Semiglazov V, Krasnozhon D et al. Comparison of EP2006, a filgrastim biosimilar, to the reference: a Phase III, randomized, double-blind clinical study in the prevention of severe neutropenia in patients with breast cancer receiving myelosuppressive chemotherapy. Ann. Oncol. 26(9), 1948-1953 (2015). 
6 Aapro MS, Bohlius J, Cameron DA et al. 2010 update of EORTC guidelines for the use of granulocyte-colony stimulating factor to reduce the incidence of chemotherapy-induced febrile neutropenia in adult patients with lymphoproliferative disorders and solid tumours. Eur. J. Cancer 47(1), 8-32 (2011).

- The most recent European Organisation for Research and Treatment of Cancer guidelines on the use of granulocyte-colonystimulating factor (G-CSF) to reduce the incidence of chemotherapy-induced febrile neutropenia.

7 Smith TJ, Khatcheressian J, Lyman GH et al. 2006 update of recommendations for the use of white blood cell growth factors: an evidence-based clinical practice guideline. J. Clin. Oncol. 24, 3187-3205 (2006).

- The most recent American Society of Clinical Oncology guidelines on the use of G-CSF to reduce the incidence of chemotherapy-induced febrile neutropenia.

8 Molineux G. Pegylation: engineering improved pharmaceuticals for enhanced therapy. Cancer Treat. Rev. 28(sA), 13-16 (2002).

9 Holmes FA, Jones SE, O'Shaughnessy J et al. Comparable efficacy and safety profiles of once-per-cycle pegfilgrastim and daily injection filgrastim in chemotherapy-induced neutropenia: a multicenter dose-finding study in women with breast cancer. Ann. Oncol. 13(6), 903-909 (2002).

10 Green MD, Koelbl H, Baselga J et al. A randomized double-blind multicenter Phase III study of fixed-dose singleadministration pegfilgrastim versus daily filgrastim in patients receiving myelosuppressive chemotherapy. Ann. Oncol. 14(1), 29-35 (2003).
11 Cooper KL, Madan J, Whyte S, Stevenson MD, Akehurst RL. Granulocyte colonystimulating factors for febrile neutropenia prophylaxis following chemotherapy: systematic review and meta-analysis. BMC Cancer 11, 404 (2011).

12 Weycker D, Malin J, Barron R et al. Comparative effectiveness of filgrastim, pegfilgrastim, and sargramostim as prophylaxis against hospitalization for neutropenic complications in patients with cancer receiving chemotherapy. Am. J. Clin. Oncol. 35(3), 267-274 (2012).

13 Klastersky J, Awada A. Prevention of febrile neutropenia in chemotherapy-treated cancer patients: pegylated versus standard myeloid colony stimulating factors. Do we have a choice? Crit. Rev. Oncol. Hematol. 78, 17-23 (2011).

14 Holmes FA, O'Shaughnessy JA, Vukelja S et al. Blinded, randomized, multicenter study to evaluate single administration pegfilgrastim once per cycle versus daily filgrastim as an adjunct to chemotherapy in patients with high-risk stage II or stage III/IV breast cancer. J. Clin. Oncol. 20(3), 727-731 (2002).

15 Bondarenko I, Gladkov OA, Elsaesser R et al. Efficacy and safety of lipegfilgrastim versus pegfilgrastim: a randomized, multicenter, active-control Phase 3 trial in patients with breast cancer receiving doxorubicin/docetaxel chemotherapy. BMC Cancer 13, 386 (2013).

16 Buchner A, Elsässer R, Bias P. A randomized, double-blind, active control, multicenter, dose-finding study of lipegfilgrastim (XM22) in breast cancer patients receiving myelosuppressive therapy. Breast Cancer Res. Treat. 148(1), 107-116 (2014).

17 Blackwell K, Paltuev R, Donskih R et al. Proposed biosimilar pegfilgrastim (LAEP2006) and reference pegfilgrastim for the prevention of neutropenia in patients with breast cancer: a randomized, double-blind trial. PROTECT 2: Pegfilgrastim Randomized Oncology (supportive care) Trial to evaluate comparative treatment results. Presented at: $57^{\text {th }}$ ASH Annual Meeting \& Exposition. San Diego, CA, USA, 4-8 December 2015.

- Results from PROTECT-2, one of the two pivotal registration trials with similar protocols performed for biosimilar pegfilgrastim LA-EP2006.

18 Vogel CL, Wojtukiewicz MZ, Carroll RR et al. First and subsequent cycle use of pegfilgrastim prevents febrile neutropenia in patients with breast cancer: a multicenter, double-blind, placebo-controlled Phase III study. J. Clin. Oncol. 23(6), 1178-1184 (2005).

19 Weise M, Bielsky MC, De Smet Ket al. Biosimilars: what clinicians should know. Blood 120(26), 5111-5117 (2012).

20 Gascón P, Tesch H, Verpoort K et al. Clinical experience with Zarzio ${ }^{\circledR}$ in Europe: what have we learned? Support Care Cancer 21(10), 2925-2932 (2013).

- A pooled analysis of five postapproval studies of biosimilar G-CSF (Zarzio $\left.{ }^{\circledR}\right)$ to assess the effectiveness of biosimilars are effective in real-world clinical practice.

21 Gascón P, Aapro M, Ludwig $\mathrm{H}$ et al. Treatment patterns and outcomes in the prophylaxis of chemotherapy-induced (febrile) neutropenia with biosimilar filgrastim (the MONITOR-GCSF study). Support Care Cancer. 24, 911-925 (2016).

-• Results from MONITOR-GCSF, an international, multicenter observational study of cancer patients treated with myelosuppressive chemotherapy and receiving prophylaxis with biosimilar filgrastim (Zarzio). 